\title{
Dual Medicare and Veteran Health Administration Use and Ambulatory Care Sensitive Hospitalizations
}

\author{
Mayank Ajmera, BPharm, MS ${ }^{7}$, Tricia Lee Wilkins, PharmD, MS' , and Usha Sambamoorthi, PhD ${ }^{1,2,3}$ \\ 'Department of Pharmaceutical Systems and Policy, School of Pharmacy, West Virginia University, Morgantown, WV, USA; ${ }^{2}$ Department of \\ Community Health and Preventive Medicine, Morehouse School of Medicine, Atlanta, GA, USA; ${ }^{3}$ HSR\&D Center for Healthcare Knowledge \\ Management, Veterans Administration New Jersey Healthcare System East Orange, East Orange, NJ, USA.
}

OBJECTIVE: The objective of the study is to examine the association between ambulatory care sensitive hospitalizations (ACSH) and dual Medicare/Veteran Health Administration use.

PARTICIPANTS: A nationally representative sample of Medicare beneficiaries, who participated in the Medicare Current Beneficiary Survey (MCBS).

DESIGN/MEASUREMENTS: Cross-sectional analyses $(\mathrm{N}=44,988)$ of linked fee-for-service Medicare claims and survey data from multiple years of the MCBS (20012005). Any ACSH and specific types of ACSH were measured using the list of prevention quality indicators developed by the Agency for Healthcare Research and Quality. Among veterans, dual Medicare/VHA use was defined as having inpatient or outpatient visits paid by VHA and consisted of three categories: 1) predominantVHA use; 2) some VHA use and no VHA use. Unadjusted group differences in any ACSH were tested using chisquare tests. Logistic regressions were used to analyze the association between dual Medicare/VHA use and ACSH after controlling for demographic, socio-economic status, health status, functional status, smoking status and obesity. All analyses accounted for the complex design of the MCBS.

RESULTS: Among inpatient users, 10.1\% had ACSH events for acute conditions and $15.8 \%$ for chronic conditions. Among all survey respondents, 5\% had any ACSH event. Among predominant-VHA users the rate was 4.9\% and among veterans with some VHA use it was 3.7\%. In bivariate and multivariate analyses, dual Medicare/ VHA use was not significantly associated with any ACSH. CONCLUSION: In a representative sample of Medicare beneficiaries, despite low income and health status, veterans with dual Medicare/VHA use were as likely as veterans without dual use to have any $\mathrm{ACSH}$, perhaps due to expanded healthcare access and emphasis on primary care in the VHA system.

KEY WORDS: dual utilization; ACSH; veterans; care coordination. J Gen Intern Med 26(Suppl 2):669-75

DOI: $10.1007 / \mathrm{s} 11606-011-1788-4$

(C) Society of General Internal Medicine 2011

The opinions expressed in this article are of the authors and do not reflect the views/opinions of any organization.

\section{INTRODUCTION}

Ambulatory care sensitive hospitalizations (ACSH) are associated with high financial burden of illness and are being considered as one of the accountability measures for the new Medicare accountable care organizations. ${ }^{1}$ According to the 2010 National Healthcare Quality Report, overall ACSH rates have not decreased over time and the costs were $\$ 27.1$ billion in 2007. ${ }^{2}$ Preventing ACSH represents an area in which, quality improvement is directly linked to lower healthcare expenditures. In this context, the Prevention Quality Indicators (PQIs) have been developed by the Agency for Health Care Research and Quality (AHRQ) to "flag potential problems resulting from a breakdown of health care services by tracking hospitalizations for conditions that should be treatable on an outpatient basis, or that could have been less severe if treated early and appropriately". ${ }^{3}$ Increased risk of ASCH can be as a marker of poor access to medical care. In fact, communities reporting unmet needs for medical care ${ }^{4}$ and individuals with limited access to primary care services ${ }^{5}$ have been shown to have higher rates of ACSH. In addition, it has been suggested that primary care interventions can produce cost-savings by avoiding ACSH. ${ }^{6}$ These findings highlight the need for access to comprehensive primary care services to reduce the risk of ASCH.

Even when access to primary care is equalized as in the case of veterans enrolled in the Veterans Health Administration (VHA) system, some subgroups have greater rates of ACSH. For example, in specific disease conditions such as dementia, veterans living in rural areas were more likely to have ACSH as compared to their urban counterparts. ${ }^{7}$ Although ACSH rates have declined between 2000 and 2007 among VHA users, ${ }^{8-10}$ substantial variation in ACSH rates across Veteran Integrated Services Networks existed; most networks in the east coast performed better than those on the west coast. ${ }^{9}$ However, this study did not include ACSH that could have occurred in VHA. Including both VHA and Medicare utilization is important because many veterans use both VHA and Medicare services. Based on a nationally representative sample, it has been reported that VHA users received most of their health care outside of the VHA system, paid through private insurance or Medicare. ${ }^{11}$ Among VHA users with outpatient use, 36\% were Medicare-only users, highlighting the importance of capturing Medicare utilization. ${ }^{12}$

Dual Medicare/VHA use (hereafter referred to as dual use) can favorably or unfavorably impact veterans' health outcomes. If veterans seek care from both Medicare and VHA for enhanced healthcare access and better disease management ${ }^{12}$, then dual 
use may not adversely affect health outcomes. On the other hand, with dual use, the potential for redundant care, health information loss and resultant fragmented care is present. ${ }^{13}$ There is some evidence that dual use is negatively associated with health outcomes among VHA users. Among veterans with diabetes, dual use, as measured by the proportion of Medicare/ VHA visits to total combined visits, was associated with poor glucose control. ${ }^{14}$ Other VHA research has documented increased mortality rates among dual users. ${ }^{15,16}$ However, to date studies that examined the relationship between ACSH and dual Medicare/VHA use are scarce. In contrast to the specific disease markers or mortality rates used in previous studies, ${ }^{14-16}$ ACSH are considered to be a starting point in identification of unmet community healthcare needs and are important to comprehensive health care delivery systems such as VHA.

Therefore, the primary purpose of the current study is to examine the association between ACSH and dual use among veterans using a nationally representative survey of Medicare beneficiaries. It has to be noted that the terms PQIs, preventable hospitalizations, and ambulatory care sensitive hospitalizations (ACSH) are often used interchangeably-we have chosen to use the term ACSH.

\section{METHODS}

\section{Data}

We used the Medicare Current Beneficiary Survey (MCBS), a "nationally representative sample of aged, disabled, and institutionalized Medicare beneficiaries". ${ }^{17}$ The MCBS data consists of two annually released public use files: "access to care" and "cost and use". The "access to care" files contain information relating to respondents' access to medical providers and their satisfaction with healthcare, and include beneficiaries who were enrolled in Medicare for the entire year. The "cost and use" files, in contrast, contain information on personal healthcare expenditures and payment sources for all beneficiaries who were eligible for Medicare at any time throughout the calendar year. Sources of information on expenditures and utilization for healthcare services include in-person interviews conducted every four months, as well as Medicare claims. The two data files can be used separately or (for those enrolled for the full year) in combination. The current project utilized multiple years of cost and use files. Although, the MCBS has a longitudinal design, we only used crosssectional annual data for years 2001-2005. Since study years were pooled, our sample represents person-years rather than persons. However, for ease of presentation, we use the term Medicare beneficiaries rather than person-year.

Study Sample. We restricted our sample to include communitydwelling Medicare beneficiaries with fee-for-service care. The final sample size of 44,988 person-years represented 9,068 beneficiaries in 2001; 9,179 in 2002; 9,198 in 2003; 8,842 in 2004; and 8,701 in 2005 .

\section{Key Independent Variable. Dual Medicare/Veteran Health} Administration Use

We first identified veteran status based on positive responses to specific questions on whether respondents had ever served in the armed forces during Korean conflict, Vietnam era, World War I or II and peace time. Among veterans, we then identified dual use. In the literature dual use has been identified in various ways. In one study, that used the MCBS, VHA use was defined among veterans by a threshold amount of less than $\$ 100$ paid by other sources such as Medicare, Medicaid, and private insurance. ${ }^{18}$ Other studies based on VHA data have used the proportion of VHA visits to total combined Medicare/VHA visits ${ }^{14}$, or the proportion of a veteran's VHA costs to total (Medicare and VHA) health care costs ${ }^{12}$. We adopted the visit-based approach and used the event summary files to count the total number of inpatient, outpatient, medical provider, and prescribed medication events among veterans. Veterans who had at least one inpatient hospitalization paid by VHA or greater than onethird ambulatory care visits to VHA facilities or greater than one-third of prescriptions paid by VHA were considered as predominant-VHA users. Veterans who had less than onethird ambulatory care visits or prescription medications paid by VHA were considered as having some VHA use. Veterans who did not have any VHA use were considered as non-VHA users. Thus, our VHA use variable was categorized into 4 groups: 1) predominant-VHA use; 2) some VHA use; 3) No VHA use and 4) Non-veterans.

Dependent Variable. Any Ambulatory Care Sensitive Hospitalization

We measured presence or absence of any ACSH based on the following 13 ambulatory care sensitive conditions identified by AHRQ as PQIs: 1) diabetes short-term complications; 2) diabetes long-term complications; 3 ) perforated appendicitis; 4) chronic obstructive pulmonary disease (COPD); 5) hypertension; 6) congestive heart failure; 7) dehydration; 8) bacterial pneumonia; 9) urinary infections; 10) angina without a procedure; 11) uncontrolled diabetes; 12) adult asthma; and 13) lower extremity amputations. Medicare beneficiaries who had a hospitalization for any of the abovementioned conditions in the observed calendar year were considered to have an ACSH. ACSH were identified using PQI software developed by $\mathrm{AHRQ},{ }^{19}$ in which the International classification of diseases, $9^{\text {th }}$ edition, clinical modification (ICD-9-CM) codes were used.

Other Independent Variables. Demographic variables were gender (women,men), race/ethnicity (white, African American, Latino, other), age in years (less than 55 years, 56-64, 65-69, 7074 and 75 or older), marital status (married,widowed,divorced/ separated, and other), metropolitan status (metro,non-metro), and census region. Socioeconomic status included education (less than high school, high school, some college and college), poverty status (less than 200\% Federal Poverty Line, 200-400\% and greater than 400\%), Medicaid (yes, no), private insurance (yes, no), and prescription drug coverage (yes, no).

Health status was measured by self-perceived general health (excellent, very good, good, fair, poor), smoking status (current, past, never) and body mass index (normal, overweight, obese and morbidly obese). Functional status was based on a count of Activities of Daily Living (ADL) Limitations. History of medical and mental illnesses (presence, absence) was derived from a list of self-reported conditions. Year of observation was used as an additional covariate to control for time effects. 
Table 1. Description of Study Sample Characteristics by Dual Medicare/VHA Use Medicare Current Beneficiary Survey, 2001-2005 ( $\mathrm{N}=44,988)$

\begin{tabular}{|c|c|c|c|c|c|c|c|}
\hline & \multirow{2}{*}{\multicolumn{2}{|c|}{ Total }} & \multicolumn{3}{|c|}{$\begin{array}{l}\text { Veteran Health Administration Use among } \\
\text { Veterans }\end{array}$} & \multirow[t]{2}{*}{ Non-Veterans } & \\
\hline & & & \multirow{2}{*}{$\begin{array}{l}\text { Predominant } \\
\text { Wt \% }\end{array}$} & \multirow{2}{*}{$\begin{array}{l}\text { Some } \\
\text { Wt \% }\end{array}$} & \multirow{2}{*}{$\begin{array}{l}\text { None } \\
\text { Wt \% }\end{array}$} & & \\
\hline & $\mathrm{N}$ & Wt \% & & & & $\mathrm{Wt}_{\mathrm{t}} \%$ & \\
\hline Year of Observation & & & & & & & $* * *$ \\
\hline 2001 & 9,068 & 19.0 & 16.1 & 20.0 & 20.3 & 18.8 & \\
\hline 2002 & 9,179 & 19.8 & 20.2 & 18.8 & 20.1 & 19.6 & \\
\hline 2003 & 9,198 & 20.4 & 20.3 & 19.2 & 20.5 & 20.3 & \\
\hline 2004 & 8,842 & 20.5 & 21.6 & 23.2 & 19.8 & 20.6 & \\
\hline 2005 & 8,701 & 20.4 & 21.7 & 18.8 & 19.2 & 20.6 & \\
\hline Sex & & & & & & & $* * *$ \\
\hline Women & 24,465 & 55.2 & 2.5 & 2.3 & 3.4 & 74.7 & \\
\hline Men & 20,523 & 44.8 & 97.5 & 97.7 & 96.6 & 25.3 & \\
\hline Race/Ethnicity & & & & & & & $* * *$ \\
\hline White & 35,507 & 79.8 & 82.7 & 90.5 & 89.0 & 76.9 & \\
\hline African American & 4,487 & 9.3 & 8.4 & 4.2 & 5.4 & 10.5 & \\
\hline Latino & 3,070 & 6.7 & 6.1 & 2.8 & 2.9 & 7.9 & \\
\hline Others & 1,864 & 4.2 & 2.8 & 2.4 & 2.7 & 4.7 & \\
\hline Age in Years & & & & & & & $* * *$ \\
\hline Less than 55 & 5,720 & 8.1 & 6.7 & 1.8 & 2.6 & 9.8 & \\
\hline $56-64$ & 2,205 & 6.3 & 8.7 & 2.4 & 3.7 & 6.9 & \\
\hline $65-69$ & 6,711 & 17.3 & 14.2 & 14.1 & 17.2 & 17.7 & \\
\hline $70-74$ & 8,777 & 23.1 & 23.4 & 25.7 & 26.7 & 22.0 & \\
\hline 75 , and older & 21,575 & 45.2 & 47.0 & 56.1 & 49.9 & 43.6 & \\
\hline Marital Status & & & & & & & $* * *$ \\
\hline Married & 22,118 & 52.5 & 65.7 & 75.6 & 73.5 & 45.3 & \\
\hline Widowed & 14,004 & 30.1 & 15.0 & 14.6 & 15.2 & 35.7 & \\
\hline Divorced/Separated & 4,905 & 11.1 & 15.5 & 8.8 & 8.1 & 11.6 & \\
\hline Others & 3,935 & 6.3 & 3.8 & 0.9 & 3.3 & 7.4 & \\
\hline Metro Status & & & & & & & $* * *$ \\
\hline Metro & 30,901 & 72.7 & 69.6 & 74.0 & 75.7 & 72.1 & \\
\hline Not Metro & 14,082 & 27.3 & 30.4 & 26.0 & 24.3 & 27.9 & \\
\hline Region & & & & & & & $* * *$ \\
\hline New England & 1,490 & 3.5 & 3.4 & 4.9 & 4.1 & 3.3 & \\
\hline Mid Atlantic & 6,451 & 15.8 & 13.9 & 14.4 & 15.2 & 16.2 & \\
\hline North East Central & 7,992 & 18.0 & 14.1 & 13.7 & 18.7 & 18.2 & \\
\hline North West Central & 3,287 & 6.8 & 9.8 & 13.0 & 6.6 & 6.6 & \\
\hline South Atlantic & 9,697 & 20.8 & 22.0 & 20.0 & 18.9 & 21.3 & \\
\hline South East Central & 3,557 & 7.7 & 7.6 & 4.4 & 6.7 & 8.0 & \\
\hline South West Central & 4600 & 10.1 & 12.0 & 14.3 & 11.0 & 9.6 & \\
\hline Mountain & 2,792 & 6.0 & 7.9 & 6.9 & 7.6 & 5.4 & \\
\hline Pacific & 4,241 & 9.5 & 7.1 & 7.9 & 10.7 & 9.3 & \\
\hline Puerto Rico & 881 & 1.7 & 2.2 & 0.5 & 0.5 & 2.1 & \\
\hline Education & & & & & & & $* * *$ \\
\hline No High School & 14,442 & 30.3 & 25.7 & 15.1 & 19.6 & 33.9 & \\
\hline High School & 16,381 & 36.7 & 35.8 & 38.3 & 33.3 & 37.7 & \\
\hline Some College & 6,199 & 14.3 & 18.0 & 17.9 & 16.9 & 13.2 & \\
\hline College & 7,730 & 18.6 & 20.4 & 28.7 & 30.2 & 15.1 & \\
\hline Poverty Status & & & & & & & $* * *$ \\
\hline LT $200 \% \mathrm{FPL}$ & 27,529 & 58.0 & 57.1 & 38.2 & 37.5 & 64.1 & \\
\hline $200 \%-399 \%$ FPL & 12,287 & 29.3 & 31.1 & 40.3 & 40.6 & 25.8 & \\
\hline GE $400 \%$ FPL & 5,172 & 12.7 & 11.8 & 21.5 & 21.9 & 10.1 & \\
\hline Medicaid Coverage & & & & & & & $* * *$ \\
\hline Yes & 9,466 & 17.7 & 7.0 & 4.5 & 4.8 & 22.4 & \\
\hline No & 35,522 & 82.3 & 93.0 & 95.5 & 95.2 & 77.6 & \\
\hline Private Insurance Coverage & & & & & & & $* * *$ \\
\hline Yes & 29,706 & 68.4 & 59.4 & 81.2 & 81.2 & 65.3 & \\
\hline No & 15,279 & 31.6 & 40.6 & 18.8 & 18.8 & 34.7 & \\
\hline Prescription drug Coverage & & & & & & & $* * *$ \\
\hline Yes & 33,645 & 74.9 & 43.1 & 71.0 & 78.7 & 76.4 & \\
\hline No & 11,343 & 25.1 & 56.9 & 29.0 & 21.3 & 23.6 & \\
\hline General Health & & & & & & & $* * *$ \\
\hline Excellent & 5,943 & 13.9 & 12.5 & 14.7 & 18.3 & 12.8 & \\
\hline Very good & 11,365 & 26.3 & 21.3 & 28.0 & 30.2 & 25.6 & \\
\hline Good & 14,269 & 31.6 & 31.3 & 36.1 & 31.4 & 31.6 & \\
\hline Fair & 9,081 & 19.4 & 23.0 & 13.5 & 14.1 & 20.7 & \\
\hline Poor & 4,109 & 8.8 & 11.9 & 7.7 & 6.0 & 9.3 & \\
\hline Functional Status (ADL) & & & & & & & $* * *$ \\
\hline None & 30,355 & 69.0 & 66.6 & 71.8 & 76.5 & 67.0 & \\
\hline $1-2$ & 9,571 & 20.8 & 23.4 & 21.2 & 17.3 & 21.5 & \\
\hline
\end{tabular}


Table 1. (Continued)

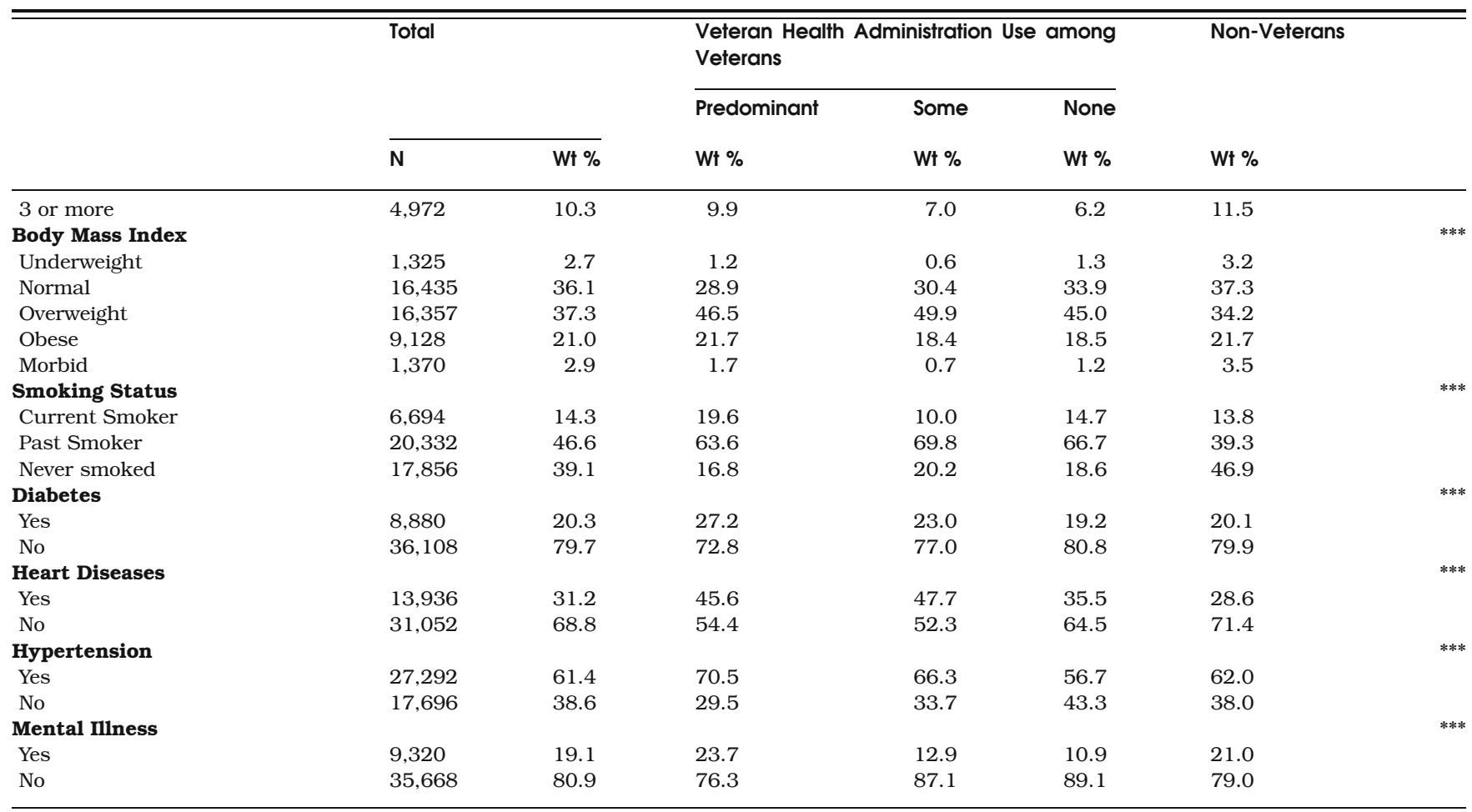

Note: Based on person years of Medicare beneficiaries, who were observed between years 2001 and 2005 and not enrolled in Medicare Health Maintenance organizations during the observation year. Asterisks represent significant relationship between Veteran Administration healthcare use and beneficiary characteristics based on chi-square tests at $p<0.001$

ADL: Activities of Daily Living; IADL: Instrumental Activities of Daily Living; FPL: Federal Poverty Line; GE: Greater than or Equal; Wt: Weighted

\section{Statistical Methods}

Chi-square tests of independence were used to determine unadjusted group differences in ACSH rates. Logistic regression on any ACSH controlled for dual use, year of observation, gender, race/ethnicity, age, marital status, metro status, region, supplemental insurance, prescription drug coverage, self-perceived general health, functional, smoking status, BMI categories, and history of mental illness. While incidence rates greater than $10 \%$ may not accurately represent relative risk, following the recommendations by Zhang and colleagues ${ }^{20}$ adjusted odds ratios (AORs) approximate relative risk due to the low rates of ACSH. Therefore, AOR and relative risk of ACSH are used interchangeably. All analyses controlled for the complex sample design of MCBS and were conducted using survey procedures with Statistical Analysis System (SAS version 9.2 Cary, North Carolina).

\section{RESULTS}

The study sample consisted of 44,957 person-years, of whom $45 \%$ were men; $80 \%$ were white; $45 \%$ were 75 years and older; $73 \%$ resided in a metropolitan area; $58 \%$ had incomes below $200 \%$ federal poverty level; $67 \%$ had no college education; and $18 \%$ had dual Medicare/Medicaid coverage.

Among veterans, of 11,759 person-years, 2,516 were predominant-VHA users, 452 had some VHA use, and 8,791 were non-VHA users. Predominant VHA users were less likely to have high income, prescription drug coverage and good health status (Table 1). For example, only $12 \%$ of predominant VHA users were in the high income group whereas $22 \%$ of veterans with no VHA use were in the high income group. Similarly,
$57 \%$ of predominant VHA users lacked prescription drug coverage, whereas $21 \%$ of veterans with no VHA use lacked the same. Higher rates of diabetes (27\% versus 19\%), heart disease (46\% versus 36\%), and hypertension ( $71 \%$ versus $57 \%$ ) were observed in veterans with predominant VHA use as opposed to their veteran counterparts with no VHA use.

Among all Medicare beneficiaries, 5\% $(\mathrm{N}=2,311)$ had any ACSH. Among those who had inpatient use, $24.3 \%$ had any ACSH; $10.1 \%$ had any acute ACSH; $15.8 \%$ had any chronic ACSH. Of the thirteen ACSH measured, the three most common hospitalizations were for congestive heart failure (7.3\%), bacterial pneumonia (6.7\%) and COPD (4.8\%) (data not shown in tabular form).

Significant subgroup differences in rates of ACSH by race/ ethnicity, age, marital status, metropolitan status and geographic region, education, income, insurance status and all health measures were noted (Table 2). When examined by dual use, the weighted average ACSH rate for veterans with predominant VHA use was $4.9 \%$; $3.7 \%$ for some VHA use; and $4.5 \%$ for veterans with no VHA use. This difference was not statistically significant.

Table 2 also presents the adjusted odds ratios (AOR) and 95\% confidence intervals (CI) from logistic regressions on presence of any ACSH. Compared to veterans with no VHA use, the risk of any ACSH was lower among veterans with predominant VHA use $[\mathrm{AOR}=0.84,95 \% \mathrm{CI}=0.64,1.11]$. However, the difference was not statistically significant. The risk of any ACSH was lower for some VHA use when compared to veterans with no VHA use; however the lower risk was also not statistically significant [AOR=0.71,95\%CI: $0.42,1.19]$. 
Table 2. Weighted Percent with Any ACSH and Adjusted Odds Ratios and $95 \%$ Confidence Intervals from Logistic Regression on Any ACSH Medicare Current Beneficiary Survey, 2001-2005

\begin{tabular}{|c|c|c|c|c|c|}
\hline ALL & Wt $\%$ & Sig & AOR & $95 \% \mathrm{Cl}$ & Sig \\
\hline \multicolumn{6}{|l|}{ Dual Medicare/ } \\
\hline \multicolumn{6}{|l|}{ VHA use } \\
\hline $\begin{array}{l}\text { Predominant } \\
\text { VHA use }\end{array}$ & 4.9 & & 0.84 & {$[0.64,1.11]$} & \\
\hline Some VHA use & 3.7 & & 0.71 & {$[0.42,1.19]$} & \\
\hline No VHA use & 4.5 & & & & \\
\hline Non-Veterans & 5.0 & & 1.01 & {$[0.84,1.21]$} & \\
\hline \multicolumn{6}{|l|}{ MCBS Year } \\
\hline 2001 & 5.0 & & & & \\
\hline 2002 & 4.8 & & 0.97 & {$[0.84,1.12]$} & \\
\hline 2003 & 5.1 & & 1.07 & {$[0.92,1.24]$} & \\
\hline 2004 & 4.9 & & 1.05 & {$[0.90,1.24]$} & \\
\hline 2005 & 4.6 & & 1.01 & {$[0.86,1.20]$} & \\
\hline \multicolumn{6}{|l|}{ Sex } \\
\hline Women & 4.9 & & 0.80 & {$[0.68,0.95]$} & $*$ \\
\hline Men & 5.0 & & & & \\
\hline Race/Ethnicity & & $* * *$ & & & \\
\hline White & 4.7 & & & & \\
\hline African American & 6.6 & & 1.03 & {$[0.86,1.24]$} & \\
\hline Latino & 4.9 & & 0.84 & {$[0.65,1.09]$} & \\
\hline Others & 4.9 & & 0.97 & {$[0.74,1.26]$} & \\
\hline Age in Years & & $* * *$ & & & \\
\hline Less than 55 Years & 3.2 & & & & \\
\hline 56-64 years & 6.6 & & 1.90 & {$[1.35,2.69]$} & $* * *$ \\
\hline $65-69$ years & 3.4 & & 2.15 & {$[1.58,2.91]$} & $* * *$ \\
\hline 70-74 years & 3.7 & & 2.44 & {$[1.83,3.26]$} & $* * *$ \\
\hline 75 or older & 6.2 & & 3.48 & {$[2.64,4.60]$} & $* * *$ \\
\hline Marital Status & & $* * *$ & & & \\
\hline Married & 4.0 & & & & \\
\hline Widowed & 6.3 & & 1.26 & {$[1.11,1.44]$} & $* * *$ \\
\hline Divorce/ & 6.4 & & 1.44 & {$[1.22,1.71]$} & $* * *$ \\
\hline \multicolumn{6}{|l|}{ Separated } \\
\hline Others & 3.1 & & 0.86 & {$[0.64,1.15]$} & \\
\hline Metro Status & & $*$ & & & \\
\hline Metro & 4.7 & & & & \\
\hline Not Metro & 5.4 & & 1.01 & {$[0.88,1.16]$} & \\
\hline Region & & $* * *$ & & & \\
\hline New England & 4.1 & & & & \\
\hline Mid Atlantic & 5.0 & & 1.14 & {$[0.85,1.53]$} & \\
\hline North East Central & 4.7 & & 1.14 & {$[0.86,1.50]$} & \\
\hline North West Central & 4.3 & & 1.20 & {$[0.82,1.74]$} & \\
\hline South Atlantic & 5.6 & & 1.19 & {$[0.90,1.59]$} & \\
\hline South East & 6.3 & & 1.23 & {$[0.87,1.73]$} & \\
\hline \multicolumn{6}{|l|}{ Central } \\
\hline South West & 5.2 & & 1.18 & {$[0.88,1.57]$} & \\
\hline \multicolumn{6}{|l|}{ Central } \\
\hline Mountain & 4.0 & & 0.99 & {$[0.73,1.33]$} & \\
\hline Pacific & 3.4 & & 0.75 & {$[0.55,1.02]$} & \\
\hline Puerto Rico & 5.8 & & 1.05 & {$[0.66,1.69]$} & \\
\hline Education & & $* * *$ & & & \\
\hline No High School & 7.3 & & 1.60 & {$[1.32,1.94]$} & $* * *$ \\
\hline High School & 4.3 & & 1.28 & {$[1.07,1.54]$} & $* *$ \\
\hline Some College & 4.5 & & 1.48 & {$[1.20,1.82]$} & $* * *$ \\
\hline College & 2.6 & & & & \\
\hline Poverty Status & & $* * *$ & & & \\
\hline Less than $200 \% \mathrm{FPL}$ & 6.2 & & & & \\
\hline 200\%-399\% FPL & 3.3 & & 1.24 & {$[1.01,1.53]$} & $*$ \\
\hline GE $400 \%$ FPL & 2.7 & & 0.95 & {$[0.78,1.17]$} & \\
\hline Medicaid Coverage & & $* * *$ & & & \\
\hline Yes & 7.8 & & 1.30 & {$[1.10,1.54]$} & $* *$ \\
\hline No & 4.3 & & & & \\
\hline Private Insurance & & $* * *$ & & & \\
\hline \multicolumn{6}{|l|}{ Coverage } \\
\hline Yes & 4.4 & & 1.15 & {$[0.99,1.34]$} & \\
\hline No & 5.9 & & & & \\
\hline Prescription Drug & & $* *$ & & & \\
\hline \multicolumn{6}{|l|}{ Coverage } \\
\hline Yes & 5.1 & & 1.10 & {$[0.95,1.27]$} & \\
\hline No & 4.3 & & & & \\
\hline
\end{tabular}

Table 2. (Continued)

\begin{tabular}{llllll}
\hline \hline ALL & W† \% & Sig & AOR & $95 \% \mathrm{Cl}$ & Sig \\
\hline General Health & & $* * *$ & & & \\
$\quad$ Excellent & 1.4 & & & & \\
Very Good & 2.1 & & 1.40 & {$[1.07,1.83]$} & $*$ \\
Good & 4.1 & & 2.46 & {$[1.85,3.27]$} & $* * *$ \\
Fair & 8.3 & & 4.58 & {$[3.45,6.08]$} & $* * *$ \\
$\quad$ Poor & 14.0 & & 7.87 & {$[5.97,10.3]$} & $* * *$ \\
$\quad$ Functional Status (ADL) & & $* * *$ & & & \\
$\quad$ None & 3.1 & & & & \\
$\quad$ 1-2 & 7.6 & & 1.59 & {$[1.42,1.79]$} & $* * *$ \\
$\quad$ or more & 11.4 & & 1.79 & {$[1.53,2.08]$} & $* * *$ \\
$\quad$ Body Mass Index & & $* * *$ & & & \\
$\quad$ Under weight & 12.4 & & 1.70 & {$[1.34,2.14]$} & $* * *$ \\
$\quad$ Normal & 5.0 & & & & \\
Overweight & 4.1 & & 0.85 & {$[0.76,0.96]$} & $* *$ \\
$\quad$ Obese & 4.9 & & 0.86 & {$[0.74,0.99]$} & $*$ \\
$\quad$ Morbid Obese & 6.3 & & 0.83 & {$[0.63,1.09]$} & \\
Smoking Status & & $* * *$ & & & \\
$\quad$ Current Smoker & 5.6 & & 1.29 & {$[1.09,1.52]$} & $* *$ \\
Past Smoker & 5.5 & & 1.39 & {$[1.23,1.57]$} & $* * *$ \\
$\quad$ Never Smoked & 3.9 & $* * *$ & & & \\
Mental Ilness & & $* * *$ & & & \\
$\quad$ Yes & 6.0 & & 0.97 & {$[0.85,1.10]$} & \\
$\quad$ No & 4.6 & & & & \\
\hline
\end{tabular}

Note: Based on 44,957 Medicare beneficiaries, who were observed between years 2001 and 2005 and not enrolled in Medicare Health Maintenance organizations during the observation year. The logistic regression also includes an intercept term not presented here. Asterisks represent significant group differences compared to the reference group based on chi-square tests and logistic regression.

ADL: Activities of Daily Living; IADL: Instrumental Activities of Daily Living; FPL: Federal Poverty Line; GE: Greater than or Equal; Wt: Weighted; AOR: Adjusted Odds Ratios; CI: Confidence Interval

*** $p<0.001$; ** $0.001<p<0.01 ; * 0.01<p<0.05$

\section{Secondary Analysis}

To ensure the robustness of the relationship between any ACSH and dual use, we analyzed the data by subgroups. These subgroups included only men and those with cardio-metabolic conditions namely, diabetes, heart disease, or hypertension. In both these sets of analyses, the findings remained consistent with the primary analyses.

\section{Controlling for Selection Bias for Dual Use}

As seen from Table 1, dual users may be a select subgroup based on poverty status, prescription drug coverage, and health status. Dual users may also differ from veterans with no VHA use by omitted variables such as VHA priority status (service-related disabilities and low-income) and unobserved variables such as veteran preferences for choosing Medicare or VHA. For example, prior literature suggests that veterans with higher VA priority status, better health status, and living near a VA facility were less likely to be dual users. ${ }^{12}$ Similarly, unobserved factors such as perceived fragmentation of care and patient satisfaction may affect dual use. ${ }^{21}$ Therefore, we controlled for the selection and reverse causality by using an instrumental variable approach. We used distance between the resident county and nearest VHA facility as an instrument in the selection equation that predicted presence/absence of dual use. The model on ACSH included all independent variables mentioned in the measures section. This analysis was restricted 
to veterans and was conducted in STATA $11 .^{22}$ The instrumental variable approach revealed that even after controlling for selection into dual use, there was no statistically significant relationship between dual use and any ACSH. Dual users were less likely to have any ACSH (parameter estimate $=-0.24, \mathrm{p}=0.20$ ); but the relationship was not statistically significant. The relationship remained consistent when testing various specifications of distance (i.e. driving distance, straight line distance, driving time, and categories of driving distance and driving time).

\section{DISCUSSION}

This paper analyzed the risk of any ACSH and dual Medicare/ VHA use among veterans for the years 2001-2005, using linked Medicare claims and a nationally representative survey of Medicare beneficiaries. Among inpatient users, nearly onequarter of hospitalizations were preventable, consistent with the published estimates. Nationwide ACSH rates using Healthcare Cost and Utilization Project data ranged from 10.1\% overall, to $16.9 \%$ among individuals whose expected source of payment was Medicare. ${ }^{23}$ However, using all Medicare beneficiaries, only $5 \%$ had an ACSH. Due to differences in denominators it should be noted that rates of ACSH based on all Medicare beneficiaries is considerably lower than those based on only hospitalizations.

Of the 13 ACSH indicators, the three most common hospitalizations were for congestive heart failure, bacterial pneumonia and COPD. These three conditions are responsible for significantly high healthcare expenditures, morbidity, mortality and productivity losses annually. ${ }^{24-29}$ As a result, hospitalizations and re-hospitalizations for these conditions have caught the attention of policy makers. Starting in 2013, the Centers for Medicare/Medicaid services will use readmissions for these conditions as an accountability measure and will reduce reimbursements to hospitals with greater than expected admission rates for these conditions. ${ }^{30}$ Future research should examine the unintended and intended consequences related to this policy.

Prior research using VHA data outlined in the introduction has reported that dual use can be associated with negative health outcomes. ${ }^{14,15}$ However, our study did not find a statistically significant association between dual VHA/Medicare use and the risk of ACSH in either bivariate or multivariate analyses. Although not statistically significant in general, dual VHA/Medicare users had a lower risk of any ACSH. As seen from our findings, veterans with predominant VHA use were poorer, lacked prescription drug coverage and had poor health than their veteran counterparts with no VHA use. Our findings from secondary analyses using instrumental approach suggest that despite having poor socioeconomic characteristics, health status and other health risk factors, dual VHA/Medicare users are as likely as veterans with no VHA use to have any ASCH. Taken together, one could suggest that even when veterans seek care from multiple systems, expanded access to healthcare through Medicare and the design of the VHA system which focuses on care coordination through primary care providers may have a protective effect. There is some evidence of improved performance of the VHA. A review of VHA system performance before and after the system-wide changes of the mid-1990s reported that 90\% of veterans received appropriate care for 9 ACSH indicators and the VHA system out-performed Medicare fee-for service on 11 of the same quality indicators. ${ }^{31}$

This study has many strong points and some weaknesses. The strengths include having the "best of both worlds" 32 by combining both Medicare claims and survey data which included variables that are not usually available in claims data (example: smoking, obesity, functional status and general health status). ACSH were extracted from Medicare claims data and based on clinical information. Non-Medicare events were derived from self-reports. Although self-reported data are subject to recall bias, the special MCBS field procedures such as interviewing respondents at relatively short intervals and verification of information including examining explanation of benefits forms help to reduce problems with recall bias and under-reporting of non-Medicare events. However, one recent study found that self-reported VHA inpatient, outpatient, and pharmaceutical events in the MCBS dataset were underreported relative to the VHA administrative databases. ${ }^{33}$ Weaknesses also include a cross-sectional study design, lack of HMO data as well as lack of diagnostic information on nonMedicare events, and small sample sizes for some conditionspecific ACSH. The study design was cross-sectional and therefore cannot be used to infer causal relationships between dual use and ACSH rates. This study does not capture use in HMOs, an important source of health care delivery for the elderly. Therefore, our results cannot be generalized to all Medicare eligible veterans. By not capturing ASCH from inpatient events that occurred in the VHA, we may have underestimated the rates of ACSH for those with dual use. However, among all hospitalizations reported by the veterans, only 3\% of hospitalizations occurred in VHA facilities and may not have a significant effect on the relationship between ASCH and dual use. As with all observational data, it is difficult to fully control for selection into VHA use.

Despite these limitations, our study provides information on hospitalizations for ambulatory care sensitive conditions using nationally representative data. The study also contributes unique information about the relationship between dual Medicare/VHA use and its association with hospitalizations for ambulatory care sensitive conditions. Our findings suggest that dual VHA/ Medicare users could be using both systems to have enhanced access in order to better manage their conditions. In addition dual users may be protected from preventable hospitalizations by the operational emphasis and organization of coordinated care in the VHA.

Acknowledgment: Dr. Sambamoorthi was partially supported by infrastructure from the Collaborative Health Outcomes Research of Therapies and Services (CoHORTS) Grant (1 P20 HS 015390-02. The authors would like to thank Dr. Patricia Findley for providing access to the MCBS data and appreciate research assistant support provided by Mr. Sandipan Bhattacharjee and Ms. Amruta Manke. The findings and opinions reported are those of the authors and do not necessarily represent the views of any other individuals or organizations.

\section{Conflict of Interest: None disclosed.}

Corresponding Author: Mayank Ajmera, BPharm, MS; Department of Pharmaceutical Systems and Policy, School of Pharmacy, West Virginia University, Morgantown, WV 26506, USA (e-mail: majmera2@hsc.wvu.edu).

\section{REFERENCES}

1. Berwick DM. Launching accountable care organizations-the proposed rule for the Medicare shared savings program. N Engl J Med. 2011;364 (16):e32. 
2. Agency for Healthcare Research and Quality, Department of Health and Human Services. National healthcare quality report, 2010. 2011. Report No.: 11-0004.

3. Kruzikas DT, Jiang HJ, Remus D, Barrett ML, Coffey RM, Andrews R. Preventable hospitalizations: A window into primary and PreventiveCare, 2000. Agency for Healthcare Research and Quality; 2004.

4. Bindman AB, Grumbach $\mathbf{K}$, Osmond D, Komaromy M, Vranizan $\mathbf{K}$, Lurie N, et al. Preventable hospitalizations and access to health care. JAMA. 1995;274(4):305-11.

5. Eun SJ, Hong J, Lee JY, Lee J, Kim Y, Kim Y, et al. [Differences in medical care utilization rates of the disabled and the non-disabled with ambulatory care sensitive conditions]. J Prev Med Public Health. 2006;39(5):411-8.

6. Sanchez A, Luis S, Blasco S, Sempere L, Iranzo JM, Zaragoza R, et al. In: Cost-benefit analysis of an intervention program in avoidable hospitalizations. ; 2007.

7. Thorpe JM, Van Houtven CH, Sleath BL, Thorpe CT. Rural-urban differences in preventable hospitalizations among community-dwelling veterans with dementia. J Rural Health. 2010;26(2):146-55.

8. Bass E, Pracht E. The declining rate of avoidable hospitalizations in the Veterans Health Administration. J Healthc Qual. 2006;28(4):45.

9. Finegan MS, Gao J, Pasquale D, Campbell J. Trends and geographic variation of potentially avoidable hospitalizations in the veterans healthcare system. Health Serv Manage Res. 2010;23(2):66-75.

10. Prentice J, Pizer S. Waiting times and hospitalizations for ambulatory care sensitive conditions. Health Services and Outcomes Research Methodology. 2008;8(1):1387-41.

11. West AN, Weeks WB. Health care expenditures for urban and rural veterans in Veterans Health Administration care. Health Serv Res. 2009;44(5): 1718-34.

12. Hynes DM, Koelling K, Stroupe K, Arnold N, Mallin K, Sohn M, et al. Veterans' access to and use of Medicare and veterans affairs health care. Med Care. 2007;45(3):214-23.

13. Hester EJ, Cook DJ, Robbins LJ. The VA and Medicare HMOscomplementary or redundant? N Engl J Med. 2005;353(12):1302-3.

14. Helmer D, Sambamoorthi U, Shen Y, Tseng C, Rajan M, Tiwari A, et al. Opting out of an integrated healthcare system: Dual-system use is associated with poorer glycemic control in veterans with diabetes. Prim Care Diabetes. 2008;2(2):73-80.

15. Wolinsky FD, Miller TR, An H, Brezinski PR, Vaughn TE, Rosenthal GE. Dual use of Medicare and the Veterans Health Administration: Are there adverse health outcomes? BMC Health Serv Res. 2006;6:131.

16. Jia H, Zheng Y, Reker DM, Cowper DC, Wu SS, Vogel WB, et al. Multiple system utilization and mortality for veterans with stroke. Stroke. 2007;38(2):355-60.
17. Center for Medicare \& Medicaid services, limited datasets [Internet].; 2009. Available at: http://www.cms.gov/LimitedDataSets/11_MCBS. asp\#TopOfPage. Accessed June 16, 2011

18. Keyhani S, Ross JS, Hebert P, Dellenbaugh C, Penrod JD, Siu AL. Use of preventive care by elderly male veterans receiving care through the Veterans Health Administration, Medicare fee-for-service, and Medicare HMO plans. Am J Public Health. 2007;97(12):2179-85.

19. Department of Health \& Human Services. AHRQ prevention quality indicators. 2008. Report No.: 3.2.

20. Zhang J, Yu KF. What's the relative risk? A method of correcting the odds ratio in cohort studies of common outcomes. JAMA. 1998;280 (19):1690-1.

21. Stroupe KT, Hynes DM, Giobbie-Hurder A, Oddone EZ, Weinberger M, Reda DJ, et al. Patient satisfaction and use of veterans affairs versus nonveterans affairs healthcare services by veterans. Med Care. 2005;43(5):453-60.

22. StataCorp. Stata statistical software. . 2009; Release 11.

23. Statistical brief \#99; healthcare cost and utilization project (HCUP) [Internet].; 2009. Available from: www.hcup-us.ahrq.gov/reports/statbriefs/sb99. jsp. Accessed June 16, 2011

24. Bui AL, Horwich TB, Fonarow GC. Epidemiology and risk profile of heart failure. Nat Rev Cardiol. 2011;8(1):30-41.

25. National Heart Lung and Blood Institute, Department of Health and Human Services. Congestive heart failure in the united states: A new epidemic. 1996.

26. Centers for Disease Control and Prevention. Pneumonia fast facts. 2010.

27. Centers for Disease Control and Prevention. Deaths from chronic obstructive pulmonary disease. 2008. Report No.: 57.

28. File Thomas M, Marrie TJ Jr. Burden of community-acquired pneumonia in North American adults. Postgrad Med. 2010;122 (2): 130-41.

29. Dalal AA, Shah M, D'Souza AO, Rane P. Costs of COPD exacerbations in the emergency department and inpatient setting. Respir Med. 2011;105(3):454-60.

30. Axon RN, Williams Mv. Hospital readmission as an accountability measure. JAMA. 2011;305(5):504-5.

31. Jha AK, Perlin JB, Kizer KW, Dudley RA. Effect of the transformation of the veterans affairs health care system on the quality of care. $\mathrm{N}$ Engl $\mathrm{J}$ Med. 2003;348(22):2218-27.

32. Eppig FJ, Chulis GS. Matching MCBS (Medicare current beneficiary survey) and Medicare data: The best of both worlds. Spring. 1997;18 (3):211-29.

33. Jonk Y, O'Connor H, Schult T, Cutting A, Feldman R, Ripley DC, et al. Using the Medicare current beneficiary survey to conduct research on Medicare-eligible veterans. J Rehabil Res Dev. 2010;47(8):797813. 